\title{
Polysomnography Applied to Patients with Tinnitus: A Review
}

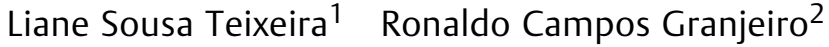 \\ Fayez Bahamad Júnior ${ }^{3}$ \\ ${ }^{1}$ Department of Otolaryngology, Universidade de Brasília, Brasília, \\ Distrito Federal, Brazil \\ 2 Sector of Auditory Health, Hospital Universitário de Brasília, Asa \\ Norte, Brasília, DF, Brazil \\ ${ }^{3}$ Faculty of Health Sciences, Universidade de Brasília, Brasília, Distrito \\ Federal, Brazil
}

Carlos Augusto Pires de Oliveira ${ }^{1}$

Int Arch Otorhinolaryngol 2018;22:177-180.

\begin{abstract}
Address for correspondence Liane Sousa Teixeira, MD, Departmento de Otolaringologia, Universidade de Brasília, Campus Universitário Darcy Ribeiro, Brasília, Distrito Federal 70910-900, Brazil (e-mail: liane_st21@hotmail.com).
\end{abstract}

\begin{abstract}
Keywords

- polysomnography

- tinnitus

- sleep

Introduction Tinnitus has been defined as an "auditory phantom perception," meaning that tinnitus results from an abnormal activity within the nervous system, in the absence of any internal or external acoustic stimulation. About 10 to $15 \%$ of the adult population is affected by tinnitus, and a relevant percentage of tinnitus sufferers experience symptoms severe enough to significantly affect quality of life, including sleep disturbances, work impairment, and, in some cases, psychiatric distress. The selfrated complaints about tinnitus focus on emotional distress, auditory perceptual difficulties, and sleep disturbances.

Objectives To evaluate the works that show sleep disorders in patients with tinnitus, and sleep disorders assessed by polysomnography.

Data Synthesis We found four studies with polysomnography to assess sleep disorders in patients with tinnitus. The first study evaluated 80 patients who were military personnel without major psychiatric disturbances, and their tinnitus was associated with noise-induced permanent hearing. The second study was a prospective, case-control, nonrandomized study of 18 patients affected by chronic tinnitus who were compared with a homogeneous control group consisting of 15 healthy subjects. The last work evaluated questionnaires mailed to patients before their initial appointment at the Oregon Health Sciences University Tinnitus Clinic between 1994 and 1997. These questionnaires requested information pertaining to insomnia, tinnitus severity, and loudness. Follow-up questionnaires were mailed to 350 patients 1 to 4 years (mean $5 \pm 2.3$ years) after their initial appointment at the clinic.

Conclusion There are few studies with polysomnography for the evaluation of patients with sleep disorders caused by tinnitus. This shows the need for more studies on this subject.
\end{abstract}

received

November 9, 2016

accepted

April 1, 2017

published online

June 16, 2017
DOI https://doi.org/

10.1055/s-0037-1603809.

ISSN 1809-9777.
Copyright $\odot 2018$ by Thieme Revinter

Publicações Ltda, Rio de Janeiro, Brazil
License terms

(ㄷ) (i) $\ominus$ (5) 


\section{Introduction}

Tinnitus has been defined as an "auditory phantom perception," meaning that tinnitus results from an abnormal activity within the nervous system, in the absence of any internal or external acoustic stimulation. Tinnitus is very common, and $\sim 10$ to $15 \%$ of the adult population is affected by it. Most studies indicate that the prevalence in adults, with a greater prevalence at higher ages, occurs during the sixth or seventh decades of life. Gender distinctions are not consistently reported across studies, but tinnitus prevalence is significantly higher in pregnant than in non-pregnant women. ${ }^{1,2}$

A relevant percentage of tinnitus sufferers experience symptoms severe enough to significantly affect quality of life, including sleep disturbances, work impairment, and, in some cases, psychiatric distress.

The severity of tinnitus varies from an occasional awareness of a noise ( ringing, hissing, buzzing, roaring, clicking, or rough sounds, for example) in one or both ears, to an unbearable sound that drives some persons to contemplate suicide. People with similar psychoacoustic descriptions of tinnitus may differ radically in their level of annoyance and sense of its impact on daily life. Epidemiologic data reveal that approximately one fourth of persons with tinnitus are discomforted by it, whereas the remaining three fourths experience the condition without significant symptoms. ${ }^{3,4}$ There are reports that tinnitus has led to suicide, although it is probable that other psychological factors play a more important role in suicide than tinnitus. ${ }^{5}$

Tinnitus, as a result of sudden sensorineural hearing loss (SSHL), is widely believed to be a neurological phenomenon. Increased bursting and synchronicity in the central auditory structures, as a result of cochlear pathology, have been implicated in the generation of tinnitus. A prevailing theory is that tinnitus is a reaction to reduced information in the frequency bands damaged by hearing loss. This has led researchers to investigate methods of acoustically stimulating the damaged frequency bands as a means of alleviating symptoms. ${ }^{6,7}$

Tinnitus can result in mood changes, fear, anxiety, depression, sleep disorders, concentration problems, and several other psychological/emotional issues, which can severely affect a person's quality of life. As a consequence, more and more patients find their way to the Otorhinolaryngology-Head and Neck Surgery Department with tinnitus as their main complaint. Therefore, an extensive audiologic assessment is necessary as part of the diagnosis and in order to prescribe the proper therapy. ${ }^{8}$

The tinnitus sensation may occur before and during sleep because of the masking effect of reducing outside noise, resulting in an increased awareness of the tinnitus sensation. ${ }^{9}$

Several studies have found that sleep was listed as the most difficult action in tinnitus patients. Insomnia was classified as the main complaint in $50 \%$ of severe tinnitus patients, and the severity of tinnitus correlated with the incidence of sleep disturbances. ${ }^{9,10}$

The prevalence of sleep disturbances in tinnitus patients varies from 25 to $77 \%$ (overall prevalence of sleep disturbances). ${ }^{11}$

Sleep disorders affect a great percentage of the population. The diagnosis of these disorders is usually made by polysomnography. ${ }^{12}$ Sleep complaints are among the most prominent complaints accompanying tinnitus, but objective data are rare.

The objective of this review is to identify studies that evaluated tinnitus using polysomnography.

\section{Review of Literature}

The search strategy employed in this literature review was guided by the combination of two descriptors indexed in the Medical Subject Headings (MeSH): tinnitus and polysomnography. Therefore, we performed a systematic review using the same descriptors in the PubMed library, and analyzed all articles published. The last manual search conducted in electronic databases occurred in May 2016.

The entire search process, the selection of the articles and data extraction, was conducted in pairs. After the search, the articles were selected based on their titles and abstracts. Subsequently, the texts of all articles were obtained in full.

A systematic literature search was performed using the Medline database, including hand-searching reference lists from original articles. We identified four studies.

Each study was reviewed by two separate authors (L.S.T and I.B.O) who independently screened abstracts and titles using the following inclusion criteria:

i) articles written in the English;

ii) studies that used polysomnography to assess patients with tinnitus;

iii) studies on the topic of tinnitus and polysomnographic assessment.

During the investigation of the articles, we found four studies that used polysomnography in the analysis of patients with chronic tinnitus. The results are shown in - Table 1.

\section{Discussion}

Few studies used polysomnography to analyze the sleep of patients with tinnitus. Aside from being a difficult exam, it is considered expensive. As a result, the vast majority of studies use only sleep questionnaires because of the ease of administration and lower cost.

Hébert et $\mathrm{al}^{13}$ prospectively examined the subjective and objective sleep parameters of this patient population to determine the differences in sleep disturbances associated with chronic tinnitus compared with matched controls. A total of 44 subjects ( 22 with tinnitus and 22 controls without tinnitus), unselected with respect to sleep complaints, participated in this study. The analysis involved 1-week sleep diaries, subjective sleep questionnaires, and 1 night of polysomnographic (PSG) assessment. Compared with the matched controls, the tinnitus group showed lower subjective sleep quality as measured with the Pittsburgh Sleep Quality Index (PSQI) and the sleep diaries, but no significant difference in objective polysomnographic sleep parameters (that is, sleep latency, efficiency). However, the quantitative non-rapid eye movement sleep analysis revealed lower spectral power in the delta frequency band in the tinnitus 
Table 1 Characterization of the studies

\begin{tabular}{|l|l|l|l|}
\hline Author & Number of individuals & Study design & Instrument \\
\hline Hébert et al $^{13}$ & $\begin{array}{l}22 \text { with tinnitus and } 22 \text { controls } \\
\text { without tinnitus }\end{array}$ & Prospective study & $\begin{array}{l}\text { Pittsburgh Sleep Quality Index } \\
\text { (PSQI) }\end{array}$ \\
\hline Attanasio et al $^{14}$ & $\begin{array}{l}18 \text { with chronic tinnitus and a } \\
\text { control group consisting of } \\
15 \text { healthy subjects. }\end{array}$ & $\begin{array}{l}\text { Prospective, case-control, } \\
\text { nonrandomized study }\end{array}$ & Tinnitus Handicap Inventory (THI) \\
\hline Folmer and Griest & $\begin{array}{l}164 \text { patients returned the follow-up } \\
\text { questionnaires }\end{array}$ & Retrospective study & $\begin{array}{l}\text { Detailed questionnaires were } \\
\text { mailed to the patients with } \\
\text { information about their medical, } \\
\text { hearing, and tinnitus histories. }\end{array}$ \\
\hline Burgos et al ${ }^{15}$ & $\begin{array}{l}10 \text { patients with tinnitus who were } \\
\text { compared with } 20 \text { patients with } \\
\text { insomnia and } 20 \text { healthy controls }\end{array}$ & $\begin{array}{l}\text { Prospective, } \\
\text { case-control study }\end{array}$ & Beck Depression Inventory (BDI) \\
\hline
\end{tabular}

group compared with the controls, and this decrease was correlated with subjective sleep complaints (the lower the delta spectral power, the greater the complaints). This is the first report of an electrophysiological correlate of sleep difficulties supportive of subjective sleep complaints in the tinnitus population.

Attanasio et al $^{14}$ evaluated the sleep architecture and its possible alterations in chronic tinnitus patients, and investigated any possible correlation between sleep architecture modifications and tinnitus perception, adaptation, and the degree of discomfort in these patients. They performed a prospective, case-control, nonrandomized study, in which 18 patients affected by chronic tinnitus were compared with a homogeneous control group consisting of 15 healthy subjects. The experimental group was enrolled at the Tinnitus Ambulatory at Policlinico Umberto I's Department of Sensory Organs, and the control group was composed of voluntary subjects. A full overnight polysomnography was performed on both groups. The tinnitus patients answered two questionnaires: the Tinnitus Handicap Inventory (THI), and a questionnaire concerning their subjective sleep quality, tinnitus intensity before bedtime, tinnitus intensity at remembered nocturnal wake-up periods, and tinnitus intensity at morning wake-up. The controls completed only the sleep quality questionnaire. All tinnitus patients had a statistically significant alteration in sleep stages. The average percentage of stage $1+$ stage 2 was $85.4 \% \pm 6.3$, whereas, in the control group, the average percentage of stage $1+$ stage 2 was $54.9 \pm 11.2(p<0.001)$. Stages 3 and 4 and rapid eye movement (REM) sleep were lacking in all tinnitus patients with an average percentage of $6.4 \pm 4.9$ of REM sleep, and $6.4 \pm 4.9$ of stages $3+4$. The control group showed an average percentage of $21.5 \pm 3.6$ of REM sleep and $21.5 \pm 3.6$ of stages $3+4(p<0.001)$. No correlation was found between the decrease in REM and the increase in the THI score in the tinnitus group $(r=0.04)$. However, a mild correlation was found between the increase in light sleep (stage $1+$ stage 2 ) and the THI score reported by the tinnitus group. Therefore, patients with light sleep report a higher THI score $(r=0.4)$.

Folmer and Griest ${ }^{10}$ investigated the effects of insomnia on tinnitus severity, and determined how this relationship may evolve with the passage of time. Questionnaires were mailed to patients before their initial appointment at the Oregon Health Sciences University Tinnitus Clinic between 1994 and 1997. These questionnaires requested information pertaining to insomnia, tinnitus severity, and loudness. During their initial appointment, the patients received counseling, education, and reassurance about tinnitus, audiometric and tinnitus evaluations, and treatment recommendations. Follow-up questionnaires were mailed to 350 patients 1 to 4 years (mean $5 \pm 2.3$ years) after their initial appointment at the clinic. A total of 174 patients ( 130 men, 44 women; mean age 55.9 years) returned the follow-up questionnaires. Although many of these patients improved in both sleep interference and tinnitus severity, a significant number (43) reported on the follow-up questionnaire that they continued to have difficulty sleeping. The reported loudness and severity of tinnitus were significantly greater for this group than for the groups of patients who reported that they never or only sometimes have difficulty sleeping. The relationship between sleep disturbance and tinnitus severity became more pronounced with the passage of time. Insomnia is associated with greater perceived loudness and severity of tinnitus. These findings underscore the importance of identification and successful treatment of insomnia for patients with tinnitus.

A final study looked at the sleep stages and other variables in 10 patients with tinnitus who were compared with 20 patients with insomnia and 20 healthy controls. As to the continuity of sleep, the patients with tinnitus showed decreased sleep efficiency, shorter sleep time, and greater number of awakenings, and the results were significant. The patients with tinnitus had a lower percentage of stage 2 , higher percentage of stage 1 , and lower latency for REM sleep compared with the controls, but without statistical significance. ${ }^{15}$

The studies were important to evaluate the stages of sleep in an objective way, especially the stage of REM sleep, considered the phase of restful sleep.

\section{Final Comments}

Polysomnography has proved to be a useful examination in the investigation of patients with chronic tinnitus. 
The work shows that few studies have assessed tinnitus by polysomnography. The vast majority of studies evaluated the sleep of patients with tinnitus only with questionnaires.

We believe that more studies using polysomnography for the objective evaluation of tinnitus are necessary.

\section{References}

1 Henry JA, Dennis KC, Schechter MA. General review of tinnitus: prevalence, mechanisms, effects, and management. J Speech Lang Hear Res 2005;48(05):1204-1235

2 Gurr P, Owen G, Reid A, Canter R. Tinnitus in pregnancy. Clin Otolaryngol Allied Sci 1993;18(04):294-297

3 Meyerhoff WL, Cooper JC. Tinnitus. In: Paparella MM, ed. Otolaryngology. 3d ed. Philadelphia: Saunders; 1991:1169-75. 2

4 Schleuning AJ II. Management of the patient with tinnitus. Med Clin North Am 1991;75(06):1225-1237

5 Blakley BW. Tinnitus Treatment Trends. Otol Neurotol 2016;37(07): 991-995

6 Jastreboff PJ. Phantom auditory perception (tinnitus): mechanisms of generation and perception. Neurosci Res 1990;8(04):221-254

7 Noreña AJ, Eggermont JJ. Changes in spontaneous neural activity immediately after an acoustic trauma: implications for neural correlates of tinnitus. Hear Res 2003;183(1-2):137-153
8 Gilles A, Goelen S, Van de Heyning P. Tinnitus: a cross-sectional study on the audiologic characteristics. Otol Neurotol 2014;35(03): 401-406

9 Riga M, Papadas T, Werner JA, Dalchow CV. A clinical study of the efferent auditory system in patients with normal hearing who have acute tinnitus. Otol Neurotol 2007;28(02):185-190

10 Folmer RL, Griest SE. Tinnitus and insomnia. Am J Otolaryngol 2000;21(05):287-293

11 Fioretti AB, Fusetti M, Eibenstein A. Association between sleep disorders, hyperacusis and tinnitus: evaluation with tinnitus questionnaires. Noise Health 2013;15(63):91-95

12 Somers VK, White DP, Amin R, et al. Sleep apnea and cardiovascular disease: an American Heart Association/American College of Cardiology Foundation Scientific Statement from the American Heart Association Council for High Blood Pressure Research Professional Education Committee, Council on Clinical Cardiology, Stroke Council, and Council on Cardiovascular Nursing. J Am Coll Cardiol 2008;52(08):686-717

13 Hébert S, Fullum S, Carrier J. Polysomnographic and quantitative electroencephalographic correlates of subjective sleep complaints in chronic tinnitus. J Sleep Res 2011;20(1 Pt 1):38-44

14 Attanasio G, Russo FY, Roukos R, Covelli E, Cartocci G, Saponara M. Sleep architecture variation in chronic tinnitus patients. Ear Hear 2013;34(04):503-507

15 Burgos I, Feige B, Hornyak M, et al. Chronic tinnitus and associated sleep disturbances. Somnologie (Berl) 2005;9(03):133-138 